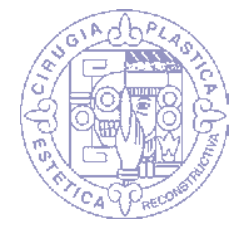

Artículo editorial

dx.doi.org/10.35366/CP191A

Estimados Compañeros Cirujanos Plásticos:

$\mathrm{N}$ o hay duda de que la evolución en nuestra especialidad nos ha llevado a buscar mejores alternativas para tratar a nuestros pacientes, así como se han producido cambios y evolución para lograr mejoría en las técnicas quirúrgicas, el cirujano plástico ha buscado dentro de sus premisas fundamentales el proporcionar seguridad total a sus pacientes. Durante muchos años, la Asociación Mexicana de Cirugía Plástica Estética y Reconstructiva ha promovido dentro de sus objetivos principales el actuar siempre en pro de la seguridad de sus pacientes. Es por ello que se formó un Comité de Seguridad constituido por múltiples miembros de nuestra asociación, el cual ha venido trabajando arduamente año con año en conjunto con múltiples mesas directivas en pro de la salud y beneficio de nuestros pacientes y de la cirugía plástica mexicana.

Gracias a este trabajo por parte del comité de seguridad se han logrado avances importantes, poniendo en alto el nombre de la cirugía plástica mexicana y de nuestra asociación a nivel nacional e internacional por los múltiples trabajos elaborados. Parte del trabajo realizado durante todos estos años ha sido recopilado y plasmado en este compendio, convirtiéndose en un tributo y reconocimiento a todos aquéllos que han trabajado por el bien de nuestra asociación, y por el bien de los pacientes que son atendidos por los cirujanos plásticos mexicanos. La Asociación Mexicana de Cirugía Plástica Estética y Reconstructiva es ya un ejemplo a seguir por múltiples asociaciones de cirugía plástica a nivel mundial, todo ello gracias a esta ardua labor tan desinteresada que han llevado a cabo todos los miembros de este importante comité de seguridad. Felicitamos a todos sus integrantes y al comité en pleno por este trabajo que han desarrollado con el único compromiso de servir a los pacientes y por ser ejemplo de honestidad y fidelidad hacia la cirugía plástica mexicana.

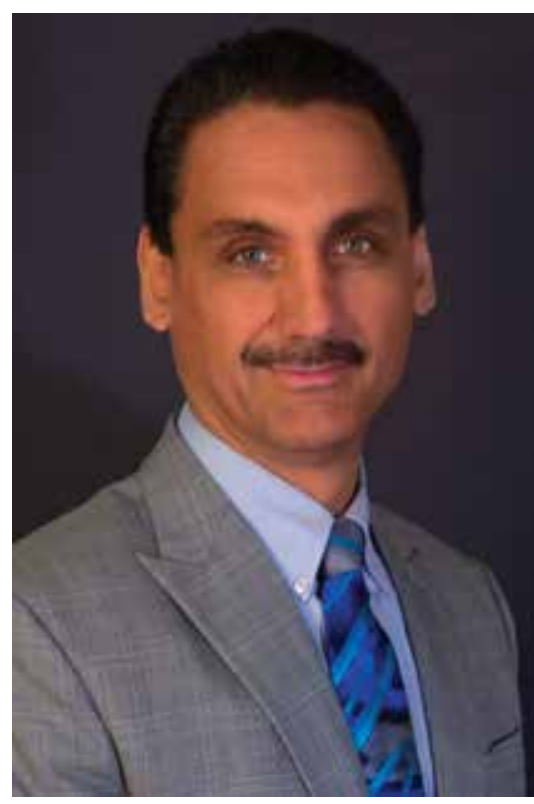

Atentamente

Dr. Lázaro Cárdenas Camarena Presidente AMCPER

El autor de este artículo 


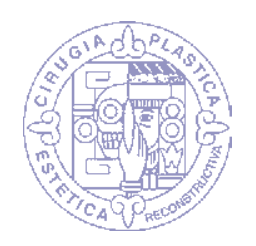

$M$ i nombre es Guillermo Ramos Gallardo, soy cirujano plástico certificado miembro del comité de seguridad de nuestra asociación. Durante cinco años he colaborado con el comité de seguridad, lo cual me enorgullece; a partir de marzo de este año seré nombrado coordinador.

Durante mi estancia en el comité he podido ver el trabajo de muchos miembros, en conjunto hemos hecho análisis de casos y peritajes. Hemos participado en la realización de encuestas, lo cual nos ha ayudado a conocer con mayor exactitud lo que pasa en el interior de la cirugía plástica mexicana, la última de ellas publicada en la revista Aesthetic Plastic Surgery, lo que me dio mucho gusto porque podemos generar una opinión científica sobre una recomendación que pareciera lógica, como no combinar la atención obstétrica con nuestra especialidad, lo cual se demostró con números. La referencia es la siguiente para quien desee buscarlo: Is Plastic Surgery Combined with Obstetrical Procedures Safe? R Iribarren-Moreno, J Cuenca-Pardo, G Ramos-Gallardo Aesthetic Plastic Surgery 2019; 43 (5), 1396-1399.

También he podido participar con las nuevas generaciones, no sólo en coordinación de eventos sino en proyectos de investigación que sirvieron como tesis y posteriormente publicación de temas tan polémicos como el embolismo graso asociado a infiltración de grasa. Sin duda, no sólo el aspecto académico ha sido muy bueno sino la oportunidad de encontrar profesionales con los mismos intereses.

Los eventos académicos en los que he participado como miembro del comite han sido experiencias inolvidables, además de sentirme orgulloso de representar a mi país y asociación en un evento tanto internacional como local. Recientemente, pude asistir a Atlanta a un evento de mama organizado por la Southeastern Society of Plastic Surgery de ese país, después asistí a un evento en Guanajuato organizado por el Colegio de Cirujanos Plásticos de Guanajuato donde, guiados por el presidente Gustavo Jiménez, hicieron un excelente intereses que declarar. no tiene conflicto de evento que en calidad de ponencias no tenía nada que pedir al de Emory.

Actualmente, estamos trabajando en líneas de investigación con resultados muy interesantes próximos a presentarse en nuestros eventos académicos, como las visitas que hicimos ocho fábricas de implantes mamarios o el registro que llevamos de estudio de cápsulas con seroma crónico en el país.

Creo que como generación nos toca aprovechar el legado de los que se nos adelantaron en el camino, profesores con una presencia extraordinaria en nuestra especialidad, pero que hoy en día el relevo nos toca a nosotros para defensa de nuestra especialidad dentro y fuera de México. Sin duda día a día veo que se cuenta con el capital humano dentro de nuestro gremio para salir adelante en un mundo cada vez más globalizado y conectado.

Agradezco a la Mesa Directiva encabezada por el Dr. Lázaro Cárdenas la oportunidad de colaborar en mi especialidad, y a ustedes el apoyo de participar en nuestras sesiones, contestar encuestas o responder en caso de alguna duda con algún caso clínico.

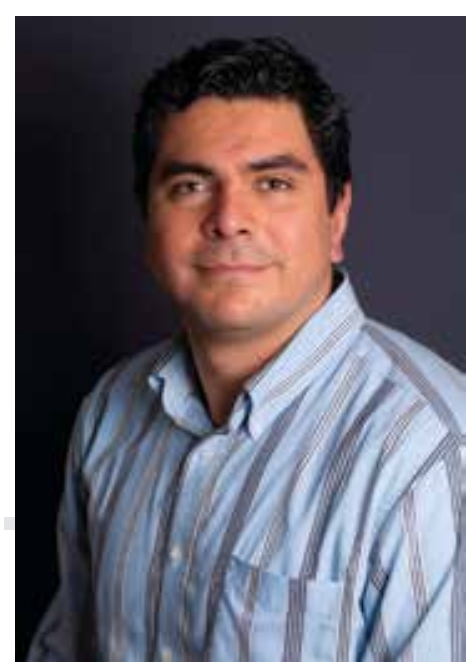

Dr. Guillermo Ramos Gallardo

Miembro del Comité de Seguridad y Coordinador de Nuevas Generaciones de AMCPER 
L a seguridad de una Cirugía Plástica siempre ha sido el principal objetivo de cualquier cirujano plástico durante sus procedimientos. Sin embargo, día a día el cirujano se enfrenta a mayores retos, tales como las expectativas irreales de las pacientes, su situación económica o el antecedente de haber sido tratadas por otros profesionales de la salud ajenos a la especialidad. Esto puede crear una situación muy delicada, ya que, al encontrarse con alguna complicación, tienen la expectativa de que al acudir ante un verdadero especialista éste le solucionará el problema de manera absoluta, sin secuelas, de forma inmediata y sobre todo que esté dentro de su presupuesto. Ello aunado a la idea de que ahora sí se cubrirán todas las expectativas iniciales sin tomar en cuenta que después de una complicación ya nada es igual.

Nosotros como especialistas expertos en la materia sabemos que la oportunidad de oro es la primera cirugía. No omito mencionar que si decidimos atender a una paciente con estas características corremos el riesgo de que olvide quién le produjo la lesión y que admita su error de haber acudido con este tipo de personajes por falta de información y cultura médica; lo cual provocará que en esta ocasión sí investigue quién es el experto y, por lo tanto, quiera atribuir que todo es responsabilidad del cirujano plástico. Así que aunque no haya complicaciones, la paciente puede quedar insatisfecha con nuestro trabajo, y más aún si llega a ocurrir una complicación. Este panorama se convierte en una situación muy delicada que terminará en un largo proceso legal, que si bien puede terminar de manera satisfactoria para el cirujano plástico, no evitará la fatiga emocional ni el desgaste de la imagen ante la población como consecuencia de la frágil posición en que nos coloca la presencia de las redes sociales.

Conscientes y entendiendo todas y cada una de estas situaciones, así como los factores condicionantes de ellas, el comité médico legal perteneciente a la división operativa ha trabajado en diferentes vertientes para mejorar la protección de todos los cirujanos plásticos de México. En primer lugar ha habido una coordinación con el comité de seguridad para garantizar que llegue a todos la información que se debe tomar en cuenta para evitar situaciones de riesgo y complicaciones en las pacientes. Se ha insistido también en un correcto protocolo de estudio de la paciente así como el exhaustivo llenado del expediente clínico. También se ha trabajado con el comité científico en la elaboración de programas académicos completos para mejorar el aspecto académico y actualizar conceptos que día a día son muy dinámicos.

Asimismo, en el comité médico legal se ha asesorado a un importante número de colegas que han necesitado apoyo legal con opiniones y dictámenes periciales en los casos que han transitado por un proceso médico legal. Casos en los que, además de ser importante llegar a la verdad científica, han sido útiles para que el comité de seguridad emita recomendaciones de seguridad a la membresía.

En otra vertiente de la búsqueda de la protección de la salud de la población y con ello garantizar lo estipulado en el artículo $4^{\circ}$ Constitucional, una actividad trascendental ha sido la defensa de nuestra especialidad. En este sentido hemos propuesto un importante número de iniciativas que han terminado en ser leyes ya publicadas, e incluso hemos detenido varias iniciativas impulsadas por grupos de profesionistas que buscaban desbloquear candados legales actuales, ya que de haber sido leyes, esas iniciativas hubieran hecho más vulnerable la salud de la población que en los últimos años se ha visto expuesta por este fenómeno internacional que representa el intrusismo médico.

Todo este esfuerzo que se hace por parte de un importante número de colegas, quienes dedican su tiempo y esfuerzo a estas actividades del comité médico legal, es y ha sido satisfactorio porque sin importar el horario, las fechas y la práctica privada de cada uno siempre se ha buscado, además de la seguridad en sus prácticas, un bien superior que es la salud de la población, situación que ha hecho que nuestra AMCPER sea una asociación socialmente responsable.

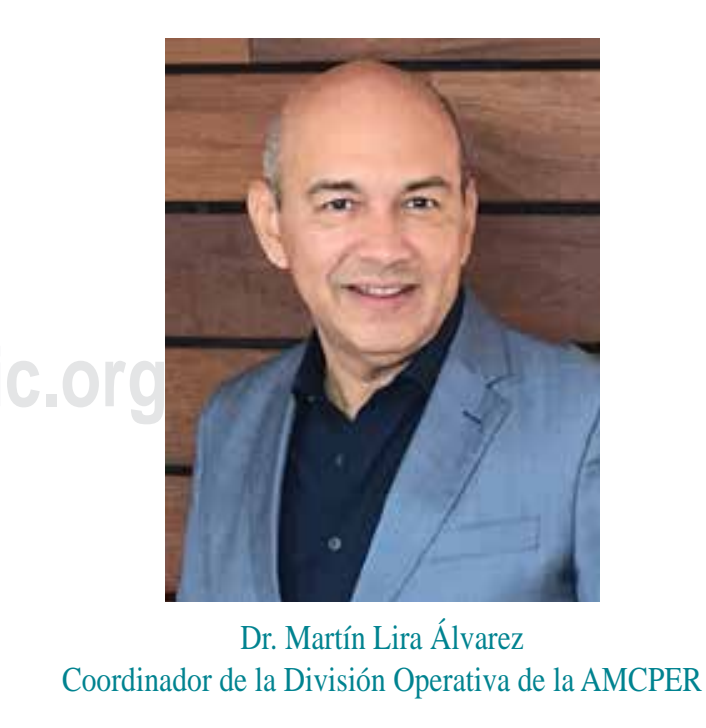

Dr. Martín Lira Álvarez

Coordinador de la División Operativa de la AMCPER no tiene conflicto de intereses que declarar. 

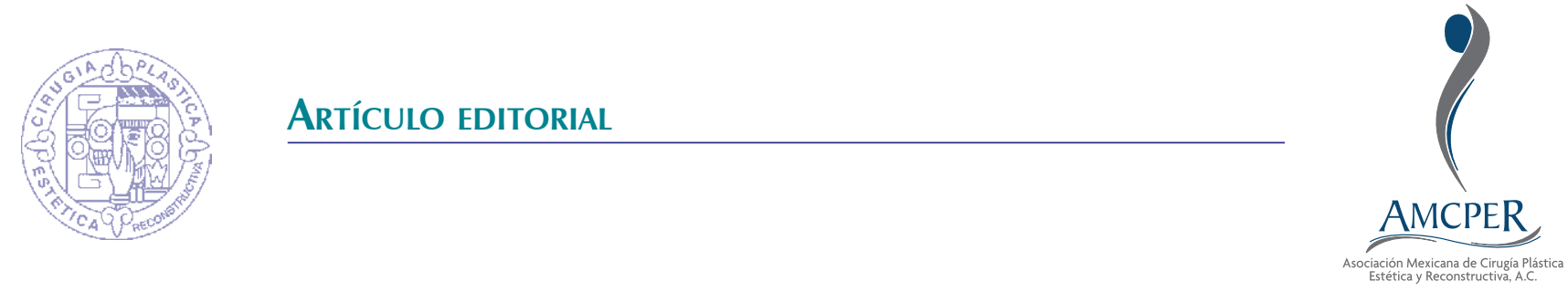

\title{
¿Se pueden evitar las complicaciones en cirugía plástica?
}

\author{
Can complications in plastic surgery be avoided?
}

\section{Dr. Jesús Cuenca-Pardo*}

— ste número de la revista está dirigido a ¿aquellos cirujanos que se preocupan por la seguridad de sus pacientes y están convencidos de que las complicaciones en cirugía se pueden evitar. Publicamos infografías, artículos de revisión sistemática MBE (Medicina Basada en Evidencia) y casos clínicos; elegimos los temas de las complicaciones más frecuentes con las que actualmente nos enfrentamos como cirujanos plásticos.

El riesgo total al que están expuestos nuestros pacientes está compuesto por cuatro factores que están relacionados con:

1. El cirujano y su equipo quirúrgico como habilidad, conocimiento y experiencia.

2. Los pacientes con enfermedades reconocidas, enfermedades ocultas o no diagnosticadas, estabilidad de sus comorbilidades, capacidades vitales y la forma de responder al trauma quirúrgico.

3. La técnica quirúrgica como la cantidad de procedimientos que realizamos en un solo tiempo a una misma paciente, el tiempo de duración de la cirugía, el grado de complejidad y agresividad de los procedimientos.

4. El hospital o clínica con recursos materiales y físicos con los que cuenta, laboratorio y terapia intensiva y personal médico de apoyo.

Si identificamos los riesgos podremos abatirlos, minimizarlos o estar preparados para cuando un evento desfavorable se presente. La mayor desgracia es cuando se presenta una complicación en forma sorpresiva y no estamos preparados para resolverla.

En el año 2008 se realizaron en México 26,000 procedimientos combinados de liposucción e infiltración grasa en los glúteos. En 2017 fueron más de 300,000 y esta tendencia al aumento trajo como consecuencia un incremento en la morbimortalidad relacionada con la lipoescultura. ${ }^{1,2}$ En el periodo 2003-2013 se detectaron 64 fallecimientos relacionados con la lipoescultura, las principales causas son: 17 (26.56\%) por embolia grasa masiva, $13(20.31 \%)$ por tromboembolia pulmonar; 20 (31.25\%) por causa desconocida y cinco $(7.81 \%)$ por perforación visceral. ${ }^{2}$ En 2014, los directivos de la Asociación Mexicana de Cirugía Plástica, Estética y Reconstructiva (AMCPER) conformaron un comité de seguridad con la finalidad de estudiar y analizar la alarmante morbimortalidad de la especialidad, y con los resultados obtenidos, emitir recomendaciones de seguridad para disminuir las complicaciones. Se integró un comité colegiado con representantes de todos los colegios y sociedades afiliadas a la AMCPER que utilizó las siguientes herramientas:

- Planeación estratégica para elaborar un diagnóstico situacional y elaborar un proyecto de seguridad a mediano y largo plazo.

- Investigación básica en cadáveres, principalmente en el estudio de la embolia grasa masiva.

- Investigación clínica para determinar la incidencia, prevalencia y factores de riesgo de las complicaciones de mayor impacto. 
- Investigación en sistemas de salud, con la finalidad de promover la seguridad como sistema con un beneficio global.

- Investigación documental con revisiones sistemáticas y metaanálisis de las complicaciones de mayor impacto en la especialidad.

- Recomendaciones de seguridad emitidas por organizaciones líderes en la seguridad de los pacientes como la Join Commission, WHO, la Alianza Internacional para la Seguridad del Paciente Quirúrgico y adaptarlas a los pacientes de cirugía plástica.

- Elaboración de dictámenes periciales de casos de fallecimiento y complicaciones, además de ayudar a la defensa de los involucrados y obtener información para determinar factores de riesgo de las principales complicaciones.

- Análisis de autopsias en diferentes servicios forenses. Solicitar los resultados de los casos de fallecimiento relacionados con procedimientos de cirugía plástica y contribuir con los estudios clínicos.

Al elaborar un diagnóstico situacional, encontramos que los principales problemas que estábamos enfrentando eran: embolia grasa

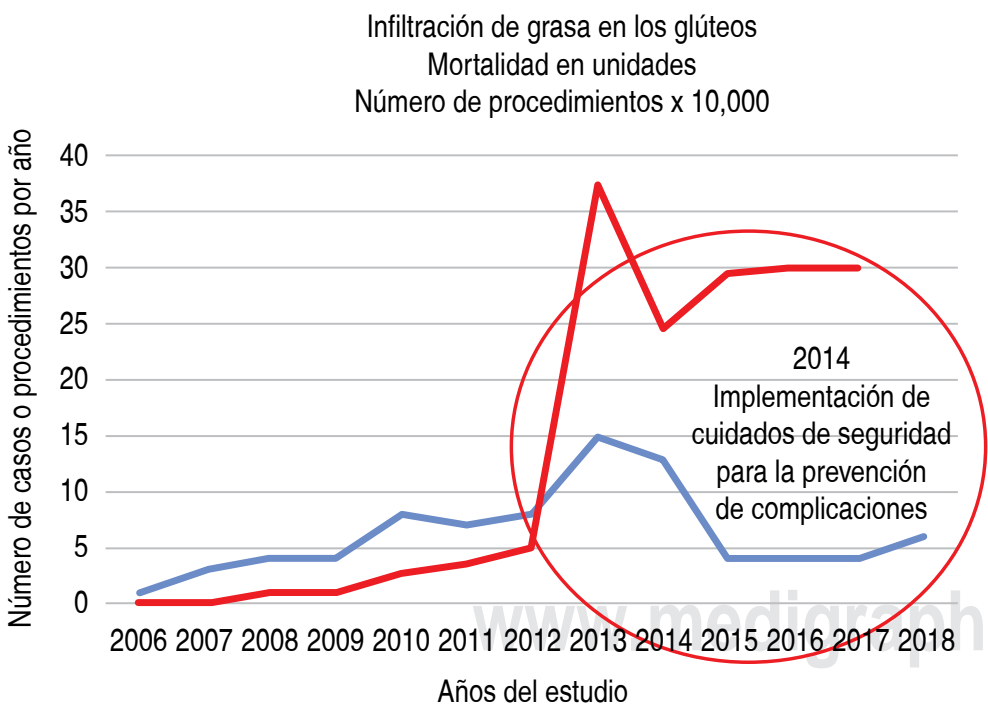

Figura 1: Con la línea azul se señala el número de fallecimientos relacionados con la lipoescultura. De continuar la tendencia era de esperar 20 fallecimientos para el año 2015. El número de procedimientos continuó siendo muy alto; sin embargo, la incidencia de mortalidad disminuyó considerablemente. Este descenso coincide con la implementación de cuidados de seguridad. masiva fulminante, tromboembolia pulmonar, perforaciones viscerales en liposucción, fallas anestésicas cardiacas, fallas con implantes mamarios y patologías relacionadas con los mismos..$^{3-7}$

La primera estrategia de seguridad que realizamos fue alertar a la comunidad con respecto a la crisis ocasionada por el aumento de la mortalidad relacionada con la lipoescultura. Esta acción creó conciencia en los directivos, profesores y en la membresía. Logramos que en todas las actividades académicas se trataran temas de seguridad, se iniciaran trabajos de investigación al respecto y que los cirujanos se hicieran más cautelosos y extremaran los cuidados en sus pacientes. ${ }^{8-14}$

La identificación de los problemas, conocer las teorías sobre las posibles causas, emitir una alerta de la crisis y crear conciencia de las complicaciones obliga a extremar los cuidados de los pacientes y comprobar las teorías causales.

Primer logro. Realizamos una regresión lineal para determinar la tendencia de la mortalidad y pronóstico; de continuar en las mismas condiciones se esperaban 20 fallecimientos en el año 2015. Con las recomendaciones de seguridad que se emitieron se logró disminuir la mortalidad: en 2015 hubo cuatro fallecimientos y en los últimos cuatro años sólo dos fallecimientos diagnosticados como embolia grasa masiva fulminante..$^{9-14}$

iLa estrategia estaba funcionando; las complicaciones se pueden evitar!

Aunque habíamos tenido un gran logro, los resultados no eran suficientes; era necesario seguir trabajando en la gestión de riesgos; analizar los factores de riesgo de los casos que aún seguían falleciendo, elaborar más recomendaciones preventivas que estuvieran basadas en la mejor evidencia médica disponible, interpretada y adaptada por expertos en la materia. Por este motivo se le dio al comité un carácter permanente, se exigió la profesionalización de sus integrantes, se agregaron asesores externos, médicos de otras especialidades y se firmaron convenios con otras especialidades con fines de seguridad del paciente quirúrgico (Figura 1, Tablas 1 y 2). 
Tabla 1: Número de casos de fallecimientos relacionados con liposucción e infiltración grasa en los glúteos.

Número de casos de fallecimientos relacionados con cirugía del contorno corporal

\begin{tabular}{ccc}
\hline & $\begin{array}{c}\text { Número de } \\
\text { muertes }\end{array}$ & Total \\
\hline 2005 & 1 & 1 \\
2006 & 1 & 2 \\
2007 & 3 & 5 \\
2008 & 4 & 9 \\
2009 & 4 & 13 \\
2010 & 8 & 21 \\
2011 & 7 & 28 \\
2012 & 8 & 36 \\
2013 & 15 & 51 \\
2014 & 13 & 64 \\
\hline Casos de mortalidad después de implementar \\
recomendaciones de seguridad \\
\hline 2015 & 4 & 8 \\
2016 & 4 & 12 \\
2017 & 4 & 18 \\
\hline 2018 & 6 & 4 \\
\hline
\end{tabular}

Además de la reducción de la morbimortalidad, la productividad del comité se ve reflejada en publicaciones en revistas internacionales y nacionales, elaboración de infografías, cursos de seguridad, participación en congresos con temas de seguridad, dictámenes periciales, sesiones mensuales y asistencia a cirujanos con complicaciones en sus pacientes (Tabla 3).

Justificación de este volumen en inglés y español dedicado a la seguridad. Es necesario que se divulgue y haga llegar la experiencia obtenida en el comité no sólo a los cirujanos plásticos de la AMCPER, sino a los cirujanos de la FILACP y de todo el mundo. Los temas y recomendaciones de seguridad tienen que ser accesibles a la mayoría de los cirujanos plásticos para su consulta y utilización. La traducción al inglés hará que una mayor cantidad de cirujanos nos lean en todo el mundo y aumente el impacto de nuestros trabajos.

Las infografías que ahora publicamos son guías rápidas y sencillas, con la información suficiente que permita al cirujano tomar una decisión de seguridad con sus pacientes. Como toda guía, requiere de revisiones y actualizaciones periódicas; asimismo, los comentarios que nos envíen los cirujanos que las utilicen nos serán de mucha utilidad para darles mayor fundamento y hacerlas más eficaces. Hemos dividido las guías en dos grandes capítulos: el primero con las infografías de los objetivos de seguridad en cirugía dictados por la OMS adaptados a la especialidad de cirugía plástica. Contamos con la aprobación de la OMS para realizar las adaptaciones y publicarlas. El segundo con recomendaciones específicas para los procedimientos más frecuentes o de mayor riesgo actual de la especialidad. Tienen su fundamento en los artículos que publicamos en este número de la revista.

Esperamos que estos trabajos sean de utilidad para todos los cirujanos plásticos en beneficio de la seguridad de nuestros pacientes; la meta es cero complicaciones y cero muertes en cirugía plástica.

\section{AGRADECIMIENTO}

Agradecimiento por participación en el diseño, elaboración y difusión de las infografías a la licenciada Liz Cárdenas y Jonathan Morales.

Tabla 2: Causas de muerte relacionadas con

liposucción e infiltración de grasa en los glúteos.

Hay una reducción importante en la incidencia de embolia grasa.

Causa de muerte periodo 2015-2018 posterior a implementar recomendaciones de seguridad

Trombosis 6

Falla cardiaca/anestésica 4

Perforación visceral

Embolia grasa

Total 6 2 18 
Tabla 3: Productividad del comité de seguridad en el periodo 2014-2019.

Productividad del comité 2014-2019

\begin{tabular}{lc}
\hline Actividad & Número \\
\hline Publicaciones Aesth Plast Surg & 5 \\
Publicaciones Plast Reconstr Surg & 5 \\
Publicaciones en Clin Plast Surg & 1 \\
Publicaciones Invest Surg & 2 \\
Publicaciones en Rev Cir Plast Iberolatinoam & 1 \\
Publicaciones en Cir Plast & 3 \\
Trabajos enviados para publicación & 7 Cir Plast; 2 Aesth Plast; 1 Plast Reconstr Surg \\
Infografías en espanol & 27 \\
Infografías en inglés & 22 \\
Cursos de seguridad & 7 \\
Participaciones en congresos & 8 \\
Sesiones de seguridad & 46 \\
Dictámenes periciales & 8 \\
Proyectos de investigación & Trombosis, riesgo total, bioseguridad de los im- \\
& plantes, patologías relacionadas con los implantes, \\
& fisiopatogenia de la embolia grasa; ácidos grasos e \\
& inflamación; factores de riesgo, incidencia y preva- \\
Asistencia a cirujanos con problemas técnicos o & lencia de las complicaciones \\
complicaciones & No cuantificado \\
\hline
\end{tabular}

\section{REFERENCIAS}

1. ISAPS Global Statistics. Disponible en: https://www. isaps.org/medical-professionals/isaps-global-statistics/

2. Cárdenas-Camarena L, Bayter JE, Aguirre-Serrano $\mathrm{H}$, Cuenca-Pardo J. Deaths caused by gluteal by lipoinjection; what are we doing wrong? Plast Reconstr Surg 2015; 136 (1): 58-66.

3. Ramos-Gallardo G, Orozco-Rentería D, MedinaZamora P, Mota-Fonseca E, García-Benavides L, Cuenca-Pardo J et al. Prevention of fat embolism in fat injection for gluteal augmentation, anatomic study in fresh cadavers. J Inv Surg 2018; 31 (4): 292-297. Doi: 10.1080/08941939.2017.1321703. http://dx.doi.org /10.1080/08941939.2017.1321703

4. Ramos-Gallardo G, Medina-Zamora P, CardenasCamarena L, Orozco-Rentería D, Duran-Vega H, MotaFonseca $\mathrm{E}$. Where does the transplanted fat is located in the gluteal region? research letter. J Invest Surg 2019; 32 (3): 228-231. Doi: 10.1080/08941939.2017.1408719. https://doi.org/10.1080/08941939.2017.1408719

5. Cárdenas-Camarena L. Embolia grasa macroscópica: el enemigo silencioso. Cir Plast Iberolaninoam 2015; 41 (4). Disponible en: http://dx.doi.org/10.4321/S037678922015000400001

6. Cárdenas-Camarena L, Durán H, Robles-Cervantes JA, Bayter-Marin JE. Critical differences between microscopic (MIFE) and macroscopic (MAFE) fat embolism during liposuction and gluteal lipoinjection. Plast Reconstr Surg 2018; 141 (4): 880-890. Doi: 10.1097/PRS.0000000000004219.

7. Durán H, Cárdenas-Camarena L, Bayter-Marin JE, Ramos-Gallardo G, Robles-Cervantes JA. Microscopic and macroscopic fat embolism: solving the puzzle with case reports. Plast Reconstr Surg 2018; 142 (4): 569e-577e. Doi: 10.1097/PRS.0000000000004810.

8. Cárdenas-Camarena L, Durán H. Improvement of the gluteal contour: modern concepts with systematized lipoinjection. Clin Plast Surg 2018; 45 (2): 237-247. Doi: 10.1016/j.cps.2017.12.005.

9. Bayter-Marin JE, Cárdenas-Camarena L, AguirreSerrano H, Durán H, Ramos-Gallardo G, RoblesCervantes JA. Understanding fatal fat embolism in gluteal lipoinjection: a review of the medical records and autopsy reports of 16 patients. Plast Reconstr Surg 2018; 142 (5): 1198-1208. Doi: 10.1097/ PRS.0000000000004904.

10. Ramos-Gallardo G, Orozco-Rentería D. Prevention of fat embolism: anatomic studies in fresh cadavers. Aesthet Surg J 2019; 39 (7): NP298-NP299. Doi: 10.1093/asj/sjz097.

11. Cárdenas-Camarena L, Andrés-Gerardo LP, Durán $\mathrm{H}$, Bayter-Marin JE. Strategies for reducing fatal complications in liposuction. Plast Reconstr Surg 
Glob Open 2017; 5 (10): e1539. Doi: 10.1097/ GOX.0000000000001539.

12. Peña W, Cárdenas-Camarena L, Bayter-Marin JE, McCormick M, Durán H, Ramos-Gallardo G et al. Macro fat embolism after gluteal augmentation with fat: first survival case report. Aesthet Surg J 2019; 39 (9): NP380-NP383. Doi: 10.1093/asj/sjz151.

13. Bayter-Marin JE, Cárdenas-Camarena L, Durán $\mathrm{H}$, Valedon A, Rubio J, Macias AA. Effects of thermal protection in patients undergoing body contouring procedures: a controlled clinical trial. Aesthet Surg J 2018; 38 (4): 448-456. Doi: 10.1093/asj/sjx155.

14. Cuenca-Pardo J, Ramos-Gallardo G, CárdenasCamarena L, Contreras-Bulnes L, Lelevier De Alvear
G. Searching for the best way to assess the risk of thrombosis in aesthetic plastic surgery; the role of the caprini/pannucci score. Aesthetic Plast Surg 2019; 43 (5): 1387-1395. Doi: 10.1007/s00266-019-01428-z.

Correspondencia:

Dr. Jesús Cuenca Pardo

Asociación Mexicana de Cirugía Plástica, Estética y Reconstructiva, A.C.

Flamencos Núm. 74,

Col. San José Insurgentes,

03900, Ciudad de México, México.

E-mail: jcuenca001@gmail.com 


\title{
Can complications in plastic surgery be avoided?
}

\author{
¿Se pueden evitar las complicaciones en cirugía plástica?
}

\section{Jesús Cuenca-Pardo, M.D.*}

$\mathrm{T}^{\mathrm{h}}$ his issue of the journal is aimed at surgeons who care about the safety of their patients and who are convinced that complications in surgery can be avoided. We publish infographics, EBM systematic review articles and clinical cases. We choose the issues of the most frequent complications that we currently face as plastic surgeons.

The total risk to which our patients are exposed is made up of four factors:

1. Factors related to the surgeon and his/her surgical team: such as, the skill, knowledge and experience.

2. Factors related to patients: diagnosed diseases, hidden or undiagnosed diseases, stability of their comorbidities, their vital abilities and response to surgical trauma.

3. Factors related to the surgical technique considering the number of procedures we perform are carried out on the same patient at one time, the duration of the surgery, the degree of complexity and aggressiveness of the procedures.

4. Factors related to the hospital or clinic: such as, the material and physical resources it has, laboratory and intensive therapy and medical support staff.

security committee of the FILACP, advisor to the security committee of the Mexican Association of Plastic Surgery.

I declare that I have no conflict of interest.

Received:

January 24, 2019

Accepted:

March 04, 2019
If we identify the risks, we can reduce them, minimize them or be prepared for when an unfavorable event occurs. The biggest misfortune is when a complication occurs suddenly, in a surprising way, and we are not prepared to solve it.

In Mexico in 2008, 26,000 combined procedures of liposuction and fat infiltration in the buttocks were performed; in 2017 there were more than 300 thousand. This growing tendency resulted in an increase in morbidity and mortality related to liposculpture. ${ }^{1,2}$ In the period 2003-2013, 64 deaths related to liposculpture were detected; the main causes are: $17(26.56 \%)$ due to massive fatty embolism; 13 (20.31\%) due to pulmonary thromboembolism; 20 (31.25\%) due to unknown causes; 5 (7.81\%) due to visceral perforation. ${ }^{2}$ In 2014, the directors of the Mexican Association of Aesthetic and Reconstructive Plastic Surgery (AMCPER) formed a Safety Committee, in order to study and analyze the referred alarming morbidity and mortality and with the results obtained issue safety recommendations, to reduce complications. A collegiate committee was integrated, with representatives from all AMCPER affiliated colleges and societies, which used the following tools:

- Strategic planning: to develop a situational diagnosis and develop a medium and longterm security project.

- Basic research on corpses, mainly in the study of massive fatty embolism.

- Clinical research: to determine the incidence, prevalence and risk factors of the complications with the greatest impact.

- Research in health systems, with the purpose of promoting safety as a system with a global benefit.

- Documentary research: systematic reviews and meta-analysis of the complications with the greatest impact on our specialty.

- Safety recommendations issued by leading organizations in patient safety such as the Joined Commission, $\mathrm{WHO}$, the International 
Alliance for surgical Patient Safety and adapting them to plastic surgery patients.

- Preparation of expert opinions in cases of death and complications; in addition to helping to defend those involved and to obtain information to determine risk factors for the main complications.

- Autopsy analysis (analysis of autopsies) in different forensic services, request the results of death cases related to plastic surgery procedures and contribute to clinical studies.

When developing a situational diagnosis, we found that the main problems we were facing were: fulminant massive fat embolism, pulmonary thromboembolism, visceral perforations in liposuction, cardiac anesthetic failures, breast implant failures and pathologies related to breast implants. ${ }^{3-7}$

The first security strategy we carried out was to alert the community the crisis caused by the increase in mortality related to liposculpture; this action created awareness among managers, teachers and members. What we achieved was that no previous reference all academic activities dealt with security issues. Research work was begun in this direction, making

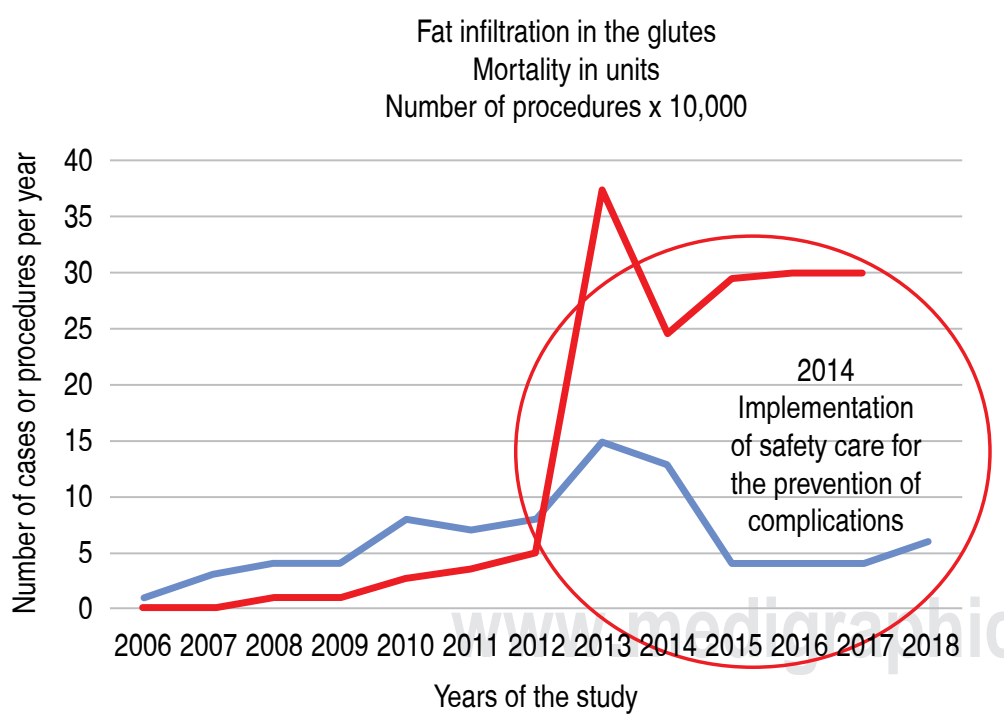

Figure 1: With the blue line, the number of deaths related to liposculpture is marked. Had the trend continued, 20 deaths were expected in 2015: The number of procedures continued to be very high; however, the incidence of mortality decreased considerably: This decrease coincides with the implementation of safety care.
Table 1: Number of cases of deaths related to liposuction and fat infiltration in the buttocks.

Number of death cases related to body contour surgery

\begin{tabular}{ccc}
\hline Year & $\begin{array}{c}\text { Number of } \\
\text { deaths }\end{array}$ & Total \\
\hline 2005 & 1 & 1 \\
2006 & 1 & 2 \\
2007 & 3 & 5 \\
2008 & 4 & 9 \\
2009 & 4 & 13 \\
2010 & 8 & 21 \\
2011 & 7 & 28 \\
2012 & 8 & 36 \\
2013 & 15 & 51 \\
2014 & 13 & 64 \\
\hline Cases of mortality after implementing safety \\
recommendations & & \\
\hline 2015 & 4 & 4 \\
2016 & 4 & 12 \\
2017 & 4 & 8 \\
2018 & 6 & \\
\hline
\end{tabular}

surgeons become more cautious and getting them to take care of their patients. ${ }^{8-14}$

The identification of the problems knowing the theories about the possible causes, issuing an alert of the crisis, creating awareness of the complications, forces plastic surgeons to take care of their patients and study the causes.

First achievement. We performed a linear regression, to determine the trend of mortality and prognosis. Had the same conditions continued, 20 deaths were to be expected in 2015 but having issued the safety recommendations, mortality was reduced; and there were 4 deaths in that year. In these last 4 years, there were only 2 deaths diagnosed as massive fulminant fat embolism. ${ }^{9-14}$

The strategy was working. Complications can be avoided! 
Although we accomplished a lot, considering our results were limited, it was necessary to continue working on risk management; analyzing the risk factors of cases of patients that continued to die, developing preventive

Table 2: Causes of deaths related to liposuction

and fat infiltration in the buttocks. There is a

significant reduction in the incidence of fatty embolism.

Cause of death period 2015-2018 after implementing safety recommendations

\begin{tabular}{lr}
\hline Thrombosis & 6 \\
Cardiac/anesthetic failure & 4 \\
Visceral perforation & 6 \\
Fat embolism & 2 \\
Total & 18 \\
\hline
\end{tabular}

recommendations based on the best medical evidence available having been interpreted and adapted by experts in this field. Because of this, the committee supervision became permanent, the professionalization of its members was required, external medical advisors of other specialties were added and agreements were signed with other specialties to guarantee surgical patient safety (Figure 1, Tables 1 and 2).

In addition to the reduction of morbidity and mortality, the productivity of the committee is reflected in: publications in international and national journals, preparation of infographics, courses based on security, participation in congresses with security issues, expert opinions, monthly sessions and support for surgeons patients with complications (Table 3).

Justification of there being a volume in English and Spanish dedicated to safety. Experience gained by the committee should be widespread and made available, not only for the

\section{Table 3: Productivity of the safety committee 2014-2019 period.}

Productivity of the Committee 2014-2019

Activity Number

Aesth Plast Surg publications
Plast Reconstr Surg publications
Clin Plast Surg publication
Invest Surg publications
Rev Cir Plast Iberolatinoam publications
Cir Plast publications
Works sent for publication

Spanish infographics

English infographics

Safety courses

Participations in congresses

Security sessions

Expert opinions

Research projects

Assistance to surgeons with technical problems or complications 
AMCPER, and the FILACP plastic surgeons, but also, for those all over the world. Safety issues and recommendations have to be accessible to plastic surgeons, for consultation and use, and the English translation will make more surgeons read about our work and increase its impact.

The infographics that we now publish are quick, simple guides, with enough information to allow the surgeon to make a decision about his or her patients. Like any guide, they require periodic reviews and updates. In a similar way, comments sent by the surgeons who use them will be very useful, to give them a better foundation and make them more effective. We have divided the guides into 2 major chapters. The first with the infographics of the safety objectives in surgery dictated by the WHO and adapted to plastic surgery. We have the approval of the $\mathrm{WHO}$ to make the adaptations and publish them. The second with specific recommendations for the most frequent or most recent procedures of the specialty are based on the articles we publish in this issue of the journal.

We hope that these works will be useful for all plastic surgeons and for the benefit of our patients' safety. Our goal is zero complications and zero deaths in plastic surgery.

\section{ACKNOWLEDGMENTS}

Acknowledgments for their participation in the design, preparation and dissemination of infographics to Ms. Liz Cárdenas and Jonathan Morales.

\section{REFERENCES}

1. ISAPS Global Statistics. Available in: https://www.isaps. org/medical-professionals/isaps-global-statistics/

2. Cárdenas-Camarena L, Bayter JE, Aguirre-Serrano $\mathrm{H}$, Cuenca-Pardo J. Deaths caused by gluteal by lipoinjection; what are we doing wrong? Plast Reconstr Surg 2015; 136 (1): 58-66.

3. Ramos-Gallardo G, Orozco-Rentería D, MedinaZamora P, Mota-Fonseca E, García-Benavides L, Cuenca-Pardo J et al. Prevention of fat embolism in fat injection for gluteal augmentation, anatomic study in fresh cadavers. J Inv Surg 2018; 31 (4): 292-297. Doi: 10.1080/08941939.2017.1321703. http://dx.doi.org /10.1080/08941939.2017.1321703

4. Ramos-Gallardo G, Medina-Zamora P, CardenasCamarena L, Orozco-Rentería D, Duran-Vega H, Mota-Fonseca E. Where does the transplanted fat is located in the gluteal region? research letter. J Invest Surg 2019; 32 (3): 228-231. Doi: 10.1080/08941939.2017.1408719. https://doi.org/1 0.1080/08941939.2017.1408719

5. Cárdenas-Camarena L. Embolia grasa macroscópica: el enemigo silencioso. Cir Plast Iberolaninoam 2015; 41 (4). Available in: http://dx.doi.org/10.4321/S037678922015000400001

6. Cárdenas-Camarena L, Durán H, Robles-Cervantes JA, Bayter-Marin JE. Critical differences between microscopic (MIFE) and macroscopic (MAFE) fat embolism during liposuction and gluteal lipoinjection. Plast Reconstr Surg 2018; 141 (4): 880-890. Doi: 10.1097/PRS.0000000000004219.

7. Durán H, Cárdenas-Camarena L, Bayter-Marin JE, Ramos-Gallardo G, Robles-Cervantes JA. Microscopic and macroscopic fat embolism: solving the puzzle with case reports. Plast Reconstr Surg 2018; 142 (4): 569e-577e. Doi: 10.1097/PRS.0000000000004810.

8. Cárdenas-Camarena L, Durán H. Improvement of the gluteal contour: modern concepts with systematized lipoinjection. Clin Plast Surg 2018; 45 (2): 237-247. Doi: 10.1016/j.cps.2017.12.005.

9. Bayter-Marin JE, Cárdenas-Camarena L, Aguirre-Serrano H, Durán H, Ramos-Gallardo G, Robles-Cervantes JA. Understanding fatal fat embolism in gluteal lipoinjection: a review of the medical records and autopsy reports of 16 patients. Plast Reconstr Surg 2018; 142 (5): 11981208. Doi: 10.1097/PRS.0000000000004904.

10. Ramos-Gallardo G, Orozco-Rentería D. Prevention of fat embolism: anatomic studies in fresh cadavers. Aesthet Surg J 2019; 39 (7): NP298-NP299. Doi: 10.1093/asj/sjz097.

11. Cárdenas-Camarena L, Andrés-Gerardo LP, Durán $\mathrm{H}$, Bayter-Marin JE. Strategies for reducing fatal complications in liposuction. Plast Reconstr Surg Glob Open 2017; 5 (10): e1539. Doi: 10.1097/ GOX.0000000000001539.

12. Peña $\mathrm{W}$, Cárdenas-Camarena L, Bayter-Marin JE, McCormick M, Durán H, Ramos-Gallardo G et al. Macro fat embolism after gluteal augmentation with fat: first survival case report. Aesthet Surg J 2019; 39 (9): NP380-NP383. Doi: 10.1093/asj/sjz151.

13. Bayter-Marin JE, Cárdenas-Camarena L, Durán H, Valedon A, Rubio J, Macias AA. Effects of thermal protection in patients undergoing body contouring procedures: a controlled clinical trial. Aesthet Surg J 2018; 38 (4): 448-456. Doi: 10.1093/asj/sjx155.

14. Cuenca-Pardo J, Ramos-Gallardo G, CárdenasCamarena L, Contreras-Bulnes L, Lelevier De Alvear G. Searching for the best way to assess the risk of thrombosis in aesthetic plastic surgery; the role of the caprini/pannucci score. Aesthetic Plast Surg 2019; 43 (5): 1387-1395. Doi: 10.1007/s00266-019-01428-z.

\section{Correspondence:}

Jesús Cuenca Pardo, M.D.

Mexican Association of Plastic Aesthetic and

Reconstructive Surgery.

Flamencos Núm. 74,

Col. San José Insurgentes,

Mexico City, Mexico.

E-mail: jcuenca001@gmail.com 